\title{
Maternal dietary betaine supplementation modifies hepatic expression of cholesterol metabolic genes via epigenetic mechanisms in newborn piglets
}

\author{
Demin Cai, Yimin Jia, Jingyu Lu, Mengjie Yuan, Shiyan Sui, Haogang Song and Ruqian Zhao* \\ Key Laboratory of Animal Physiology and Biochemistry, Nanjing Agricultural University, Nanjing 210095, \\ People's Republic of China
}

(Submitted 4 March 2014 - Final revision received 19 June 2014 - Accepted 11 July 2014 - First published online 15 September 2014)

\section{Abstract}

To elucidate the effects of maternal dietary betaine supplementation on hepatic expression of cholesterol metabolic genes in newborn piglets and the involved epigenetic mechanisms, we fed gestational sows with control or betaine-supplemented diets $(3 \mathrm{~g} / \mathrm{kg})$ throughout pregnancy. Neonatal piglets born to betaine-supplemented sows had higher serum methionine concentration and hepatic content of betaine, which was associated with significantly up-regulated hepatic expression of glycine $N$-methyltransferase. Prenatal betaine exposure increased hepatic cholesterol content and modified the hepatic expression of cholesterol metabolic genes in neonatal piglets. Sterol regulatory elementbinding protein 2 was down-regulated at both mRNA and protein levels, while 3-hydroxy-3-methylglutaryl CoA reductase (HMGCR) was down-regulated at the mRNA level, but up-regulated at the protein level, in betaine-exposed piglets. The transcriptional repression of HMGCR was associated with CpG island hypermethylation and higher repressive histone mark $\mathrm{H} 3 \mathrm{~K} 27 \mathrm{me} 3$ (histone $\mathrm{H} 3$ lysine 27 trimethylation) on the promoter, whereas increased HMGCR protein content was associated with significantly decreased expression of miR-497. Furthermore, LDL receptor was significantly down-regulated at both mRNA and protein levels in the liver of betaine-exposed piglets, which was associated with promoter CpG hypermethylation. In addition, the expression of cholesterol-27 $\alpha$-hydroxylase (CYP27 $\alpha 1)$ was up-regulated at both mRNA and protein levels, while the expression of cholesterol- $7 \alpha$-hydroxylase $(C Y P 7 \alpha 1)$ was increased at the mRNA level, but unchanged at the protein level associated with increased expression of miR-181. These results indicate that maternal betaine supplementation increases hepatic cholesterol content in neonatal piglets through epigenetic regulations of cholesterol metabolic genes, which involve alterations in DNA and histone methylation and in the expression of microRNA targeting these genes.

\section{Key words: Betaine: Epigenetic regulation: Cholesterol metabolism: Maternal diet: Piglets}

Cholesterol is an essential component of cell membranes and also serves as a precursor for life-sustaining steroid hormones and bile acids ${ }^{(1)}$. It is well known that deregulation of cholesterol metabolism contributes to obesity, diabetes and $\mathrm{CVD}^{(2,3)}$. Moreover, cholesterol is particularly essential for embryogenesis $^{(4,5)}$, and low plasma cholesterol level is usually correlated with low body weight at birth ${ }^{(6-8)}$.

Hepatic cholesterol homeostasis is maintained through the coordinated regulation of three relevant processes: biosynthesis; transportation; transformation ${ }^{(4,9)}$. In particular, sterol regulatory element-binding protein-2 (SREBP2) ${ }^{(10)}$ and 3-hydroxy-3-methylglutaryl-CoA reductase $(\mathrm{HMGCR})^{(11)}$ are key factors/enzymes for cholesterol biosynthesis, LDL receptor (LDLR) and HDL receptor (scavenger receptor class B type I (SR-BI)) are responsible for transportation ${ }^{(12)}$, while cholesterol- $7 \alpha$-hydroxylase (CYP7 $\alpha 1$ ) and cholesterol-27 $\alpha$-hydroxylase $(\mathrm{CYP} 27 \alpha 1)^{(13)}$ are main enzymes catalysing transformation to bile acids. It has been shown that hepatic cholesterol homeostasis in offspring is highly vulnerable to maternal nutrition status ${ }^{(14,15)}$, predominantly through epigenetic mechanisms such as DNA methylation, histone modification and microRNA-mediated post-transcriptional regulation ${ }^{(16)}$ Methyl donors, such as methionine or folic acid, are able to reverse the epigenetic modifications and thereby restore the behavioural or metabolic disorders in offspring caused by prenatal or neonatal insults ${ }^{(17,18)}$

Betaine, also known as betaine anhydrous or trimethylglycine, functions as a substrate for the formation of methionine, which can then be further converted to $S$-adenosylmethionine $(\mathrm{SAM})^{(19)}$. In the methionine metabolic cycle, SAM is further converted to $S$-adenosylhomocysteine by glycine $N$-methyltransferase (GNMT), thereby donating the methyl group for DNA and protein methylation catalysed by DNMT (DNA methyltransferases) and histone methyltransferases, respectively.

Abbreviations: GNMT, glycine $N$-methyltransferase; H1, histone H1; H3K27me3, histone H3 lysine 27 trimethylation; HMGCR, 3-hydroxy-3-methylglutarylCoA reductase; miRNA, microRNA; LDL-C, LDL-cholesterol; LDLR, LDL receptor; SAM, S-adenosylmethionine; SREBP2, sterol regulatory element-binding protein-2. 
Consequently, betaine supplementation may modulate gene expression through modifying epigenetic marks such as DNA and histone methylation ${ }^{(20,21)}$.

Betaine deficiency results in a series of metabolic abnormalities $^{(19)}$, while betaine supplementation in the diet can prevent obesogenic diet-induced hepatic steatosis in rodents ${ }^{(22)}$ and improve growth performance and carcass characteristics in domestic animals ${ }^{(23,24)}$. It has been observed that betaine supplementation could change cholesterol production in human subjects and animals ${ }^{(25-28)}$, yet the mechanisms remain unclear. Furthermore, although betaine is known to be critical for embryonic and fetal development ${ }^{(19)}$, the effects of betaine supplementation in maternal diet during gestation on hepatic cholesterol metabolism in neonatal offspring and the underlying mechanisms have not been investigated.

In the present study, we aimed to investigate the effects of feeding gestational sows with a betaine-supplemented diet on hepatic cholesterol metabolism in newborn piglets. To explore the potential epigenetic mechanisms underlying such effects, we studied hepatic expression of genes involved in cholesterol and methionine metabolism, and determined the status of DNA and histone methylation on the promoter of cholesterol metabolic genes, as well as the expression of microRNA targeting these genes, in the liver of neonatal piglets.

\section{Materials and methods}

\section{Animals and sampling}

A total of sixteen second-parity cross-bred sows (Landrace $X$ Yorkshire) at the age of approximately 8 months were artificially inseminated, at the observation of oestrus, with a mixture of Duroc semen samples obtained from two littermate boars. The sows were randomly divided into control and betaine groups (eight per group) after 1 week of the artificial insemination. The sows in the control group were fed a basal diet not supplemented with betaine, while those in the betaine group received a betaine-supplemented $(3 \mathrm{~g} / \mathrm{kg})$ diet throughout pregnancy. The composition of the two experimental diets is shown in Table 1. Betaine (Skystone Feed Company Limited) was added in the form of betaine hydrochloride with $98 \%$ purity. All the sows were fed three times per d at 05.00, 10.00 and 17.00 hours, and had free access to water. Immediately after parturition, newborn piglets were individually weighed and the piglets from the same litter were kept together in a warm creep area. Male piglets (one per litter) of the mean body weight of $1.59 \mathrm{~kg}$ were selected and killed before suckling. Serum samples were prepared immediately, and the liver (without the gall bladder) was taken within 20 min postmortem, snap-frozen in liquid $\mathrm{N}_{2}$ and stored at $-80^{\circ} \mathrm{C}$ until further analysis.

The experimental protocol was approved by the Animal Ethics Committee of Nanjing Agricultural University, with the project number 2012CB124703. The slaughter and sampling procedures complied with the 'Guidelines on Ethical Treatment of Experimental Animals' (2006) No. 398 set by the Ministry of Science and Technology, China.
Table 1. Composition and nutrient content of the experimental diets

\begin{tabular}{lcc}
\hline & Control & Betaine \\
\hline Ingredients (g/kg) & 370 & \\
Maize & 300 & 370 \\
Wheat & 80 & 300 \\
Bran & 170 & 80 \\
Soyabean meal & 30 & 170 \\
Lignocelluloses $_{\text {CaHPO }}$ & 20 & 30 \\
Soyabean oil $_{\text {Premix* }}^{*}$ & 8 & 20 \\
Betaine & 20 & 8 \\
Digestible energy (MJ/kg) & 0 & 20 \\
Calculated composition (\%) & 13.1 & 3 \\
Crude protein & & 13.1 \\
Crude fibre & 15 & 15 \\
Ca & 4.5 & 4.5 \\
P & 0.84 & 0.84 \\
\hline
\end{tabular}

* The premix contained the following vitamins and minerals (per kg of diet): vitamin A, $72 \mathrm{mg}$; vitamin $D_{3}, 1.5 \mathrm{mg}$; vitamin $E, 648 \mathrm{mg}$; vitamin $K_{3}, 30 \mathrm{mg}$; vitamin $B_{1}$, $30 \mathrm{mg}$; vitamin $B_{2}, 120 \mathrm{mg}$; vitamin $B_{6}, 60 \mathrm{mg}$; vitamin $B_{12}, 360 \mathrm{mg}$; niacin, $600 \mathrm{mg}$; pantothenic acid, $300 \mathrm{mg}$; folic acid, $6 \mathrm{mg}$; manganese sulphate, $1.0 \mathrm{~g}$; zinc oxide, $2.5 \mathrm{~g}$; ferrous sulphate, $4.0 \mathrm{~g}$; copper sulphate, $4.0 \mathrm{~g}$; sodium selenite, $6 \mathrm{mg}$; $\mathrm{Ca}, 150 \mathrm{~g}$; P, $15 \mathrm{~g} ; \mathrm{NaCl}, 40 \mathrm{~g}$.

\section{Determination of hepatic betaine and \\ S-adenosylmethionine content and serum methionine concentration}

For the determination of betaine concentrations, $1 \mathrm{~g}$ of frozen liver samples was prepared as described previously ${ }^{(29)}$. Betaine concentrations in the liver samples were measured with a liquid chromatography (Agilent 1200; Agilent Technologies)-MS (API 5000TM; AB Sciex) system.

Hepatic SAM content was measured using the HCB Quantitative Porcine Competitive ELISA kit (S200FC; Hermes Criterion Biotechnology) following the manufacturer's instructions. Serum concentration of methionine was determined in duplicate with an automatic amino acid analyser (L-8900; Hitachi).

\section{Measurement of cholesterol and bile acid concentrations}

Serum concentration of total cholesterol was measured using a commercial cholesterol assay kit (E1005; Applygen Technologies, Inc.). Serum concentrations of LDL-cholesterol (LDL-C) and HDL-cholesterol were measured with assay kits (006340 and 006328, respectively; Beijing BHKT Clinical Reagent Company Limited). Hepatic total cholesterol concentration was measured using a tissue total cholesterol assay kit (E1015; Applygen Technologies, Inc.) following the manufacturer's instructions. Hepatic total bile acid concentrations were determined by enzymatic colorimetric methods using a commercial kit (E003; Nanjing Jiancheng Bioengineering Institute).

\section{Real-time PCR for mRNA quantification}

For RNA extraction, approximately $200 \mathrm{mg}$ of liver samples were homogenised in $1 \mathrm{ml}$ of TRIzol reagent (Invitrogen), according to the manufacturer's protocol. RNA samples $(2 \mu \mathrm{g})$ were digested with DNase and reverse transcribed to complementary DNA using random hexamer primers 
(Promega). Diluted complementary DNA ( $2 \mu \mathrm{l}, 1: 25)$ was used in each real-time PCR assay with Mx3000P (Stratagene). Peptidylprolyl isomerase A (PPIA) was chosen as a reference gene to normalise the mRNA abundance of target genes, because it is expressed in comparable abundance to target genes and was not affected by the experimental factor. Real-time PCR data were analysed by the $2^{-\Delta \Delta C t}$ method. Primers for realtime PCR were synthesised by Generay Biotech and are listed in online supplementary Table S2.

\section{Protein extraction and Western blot analysis}

Total cellular protein and nuclear protein were extracted from $200 \mathrm{mg}$ of frozen liver samples as described previously ${ }^{(30)}$. Protein concentrations were measured with a Pierce BCA Protein Assay kit (no. 23225; Thermo Scientific). Western blot analysis of target proteins was carried out according to the protocols provided by the manufacturer. The sources of primary antibodies used in Western blot are listed in online supplementary Table S3. GAPDH or $\beta$-actin was selected as the loading controls for total cellular protein, while histone H1 (H1) was selected as the loading control for nuclear proteins.

\section{DNA methylation analysis - methylated DNA immunoprecipitation}

Genomic DNA extracted from the liver samples was sonicated to produce small fragments ranging from 300 to $1000 \mathrm{bp}$. The fragmented DNA $(2 \mu \mathrm{g})$ was heat-denatured to produce single-stranded DNA. A mouse monoclonal antibody against 5-methyl cytidine (ab10805; Abcam) was used to immunoprecipitate the methylated DNA fragments. Pretreated protein $G$ agarose beads ( $40 \mu \mathrm{l}, 50 \%$ slurry, sc-2003; Santa Cruz Biotechnology) were used to capture the immune complexes. The beads bound to immune complexes were washed to eliminate non-specific binding and resuspended in $250 \mu \mathrm{l}$ digestion buffer containing proteinase $\mathrm{K}$ to purify the immunoprecipitated methylated DNA. A small aliquot of immunoprecipitated methylated DNA was used to amplify the proximal promoter sequences of target genes by real-time PCR. A pair of negative control primers was used to amplify a promoter region absent of CPG sites as the internal control. The results of methylated DNA immunoprecipitation were calculated relative to the internal control, and expressed as the fold change relative to the mean value of the control group. The specific and negative control primers are listed in online supplementary Table S2.

\section{Chromatin immunoprecipitation assay}

Approximately $200 \mathrm{mg}$ of frozen liver samples were ground in liquid $\mathrm{N}_{2}$ and resuspended with PBS containing protease inhibitor cocktail (no. 11697498001; Roche). Cross-linking of protein and DNA was performed by adding formaldehyde to a final concentration of $1 \%$, and then the reaction was terminated with glycine $(2.5 \mathrm{~mol} / \mathrm{l})$ at room temperature. The reaction mix was centrifuged and the pellets were rinsed with PBS and homogenised in a SDS lysis buffer containing protease inhibitors. Crude chromatin preparations were sonicated to an average length ranging from 200 to $500 \mathrm{bp}$ and precleared with salmon sperm DNA-treated protein $G$ agarose beads ( $40 \mu \mathrm{l}, 50 \%$ slurry, sc-2003; Santa Cruz Biotechnology). The mixture of precleared chromatin preparations and $2 \mu \mathrm{g}$ of specific primary antibody were incubated overnight at $4^{\circ} \mathrm{C}$ (for details of antibodies, see online supplementary Table S2). A negative control was included with normal rat IgG. Protein $\mathrm{G}$ agarose beads ( $40 \mu \mathrm{l}, 50 \%$ slurry, sc-2003; Santa Cruz Biotechnology) were added to capture the immunoprecipitated chromatin complexes. Finally, DNA fragments were released from the immunoprecipitated complexes by reverse cross-linking at $65^{\circ} \mathrm{C}$ for $1 \mathrm{~h}$, and quantitative real-time PCR was used to quantify the fragments of target gene promoters with specific primers (see online supplementary Table S2) using purified immunoprecipitated DNA as the template.

\section{Quantification of microRNA}

Total RNA $(2 \mu \mathrm{g})$ treated with RNase-free DNase I (Promega) were polyadenylated by poly(A) polymerase using a poly(A) tailing kit (AM1350; Applied Biosystems), according to the manufacturer's instructions. Polyadenylated RNA was then dissolved and reverse transcribed using a poly(T) adapter. Realtime PCR was performed with SYBR Green qPCR master mix reagent (Takara) in triplicate using a microRNA (miRNA)specific forward primer and a universal reverse primer complementary to part of the poly(T) adapter sequence. U6 small-nuclear RNA (U6 snRNA) was used as a reference gene to normalise the expression of miRNA.

The sequences of all porcine miRNA were acquired from miRBase (http://www.mirbase.org/). miRNA targeting $H M G C R, L D L R, C Y P 7 \alpha 1$ and $C Y P 27 \alpha 1$ were predicted with an online miRNA prediction tool ${ }^{(31)}$. Among all the predicted miRNA, four miRNA targeting $H M G C R$, ten targeting $L D L R$, five targeting $C Y P 7 \alpha 1$ and twelve targeting $C Y P 27 \alpha 1$ were quantified by real-time PCR. The primer sequences used for miRNA analysis are listed in online supplementary Table S4.

\section{Functional validation of microRNA}

Genomic sequences of porcine miR-497 and miR-181 precursors (see online supplementary Table S5) were synthesised and inserted into the pSilencer 3.0-H1 small-interfering RNA expression vector by Invitrogen. The 3'-UTR (untranslated regions) of the porcine $H M G C R$ gene containing a conserved motif for miR-497 targeting was amplified by PCR using the primers 5 -GGGCGGGGGGACCAGCCTGCTCCCTCA- $3^{\prime}$ and $5^{\prime}$-GGAGAGAAGCACGCTCCGCTGGAGG-3'. The $3^{\prime}$-UTR of the $C Y P 7 \alpha 1$ gene containing a conserved motif for miR-181 targeting was amplified using the primers 5'-TCTGACATTACTAAACATGCCTCTA- $3^{\prime}$ and $5^{\prime}$ - TAACATTTAAAACATAATAAATTTA$3^{\prime}$. The PCR products were then cloned downstream to the pGL3-Control luciferase reporter vector (Promega) and formed the plasmids pGL3-Control/HMGCR and pGL3Control/CYP7 $\alpha 1$, respectively.

HeLa cells were cultured in Dulbecco's modified Eagle's medium containing $10 \%$ fetal bovine serum at $37^{\circ} \mathrm{C}$ in 
Table 2. Body and liver weights and hepatic total cholesterol and bile acid content, and serum cholesterol concentration in newborn piglets (Mean values with their standard errors, $n 8$ )

\begin{tabular}{|c|c|c|c|c|c|}
\hline \multirow[b]{2}{*}{ Variables } & \multicolumn{2}{|c|}{$\begin{array}{l}\text { Control group } \\
\qquad(n 8)\end{array}$} & \multicolumn{2}{|c|}{$\begin{array}{l}\text { Betaine group } \\
\qquad(n 8)\end{array}$} & \multirow[b]{2}{*}{$P$} \\
\hline & Mean & SEM & Mean & SEM & \\
\hline Body weight (kg) & 1.65 & 0.04 & 1.65 & 0.09 & 0.91 \\
\hline Liver weight $(\mathrm{g})$ & 46.08 & 1.89 & $42 \cdot 51$ & 5.73 & 0.81 \\
\hline Hepatic Tch $(\mathrm{mg} / \mathrm{g})$ & 0.94 & 0.07 & 1.29 & 0.13 & $<0.05$ \\
\hline Hepatic TBA $(\mu \mathrm{g} / \mathrm{g})$ & 30.49 & $3 \cdot 86$ & 38.27 & 4.99 & 0.24 \\
\hline Serum Tch (mmol/l) & 1.08 & 0.11 & 1.20 & 0.01 & 0.29 \\
\hline Serum LDL-C (mmol/l) & 0.50 & 0.06 & 0.58 & 0.08 & 0.43 \\
\hline Serum HDL-C $(\mathrm{mmol} / \mathrm{l})$ & 0.50 & 0.03 & 0.52 & 0.04 & 0.67 \\
\hline LDL-C:HDL-C & 0.99 & 0.09 & 1.09 & $0 \cdot 10$ & 0.47 \\
\hline
\end{tabular}

Tch, total cholesterol; TBA, total bile acids; LDL-C, LDL-cholesterol; HDL-C, HDL-cholesterol.

a $5 \% \mathrm{CO}_{2}$ incubator, and co-transfected with $100 \mathrm{ng}$ pGL3Control/HMGCR, $10 \mathrm{ng}$ Herpes simplex virus-thymidine kinase promoter (pRL-TK) plasmid and $100 \mathrm{ng}$ pSilence $3 \cdot 1$ H1-neo miR-497, or with $100 \mathrm{ng}$ pGL3-Control/CYP7 $\alpha 1$, $10 \mathrm{ng}$ pRL-TK and $100 \mathrm{ng}$ pSilencer 3.1 H1-neo miR-181, for 24 and $48 \mathrm{~h}$, respectively. Luciferase activity of the cells was measured on a luminometer according to the manufacturer's instruction using the luciferase reporter assay system (GloMax ${ }^{\circledR} 96$ Microplate Luminometer; Promega).

\section{Statistical analysis}

All data are presented as means with their standard errors Comparisons were made using independent two-tailed

(a)

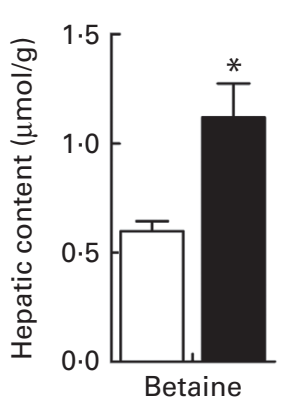

(e)

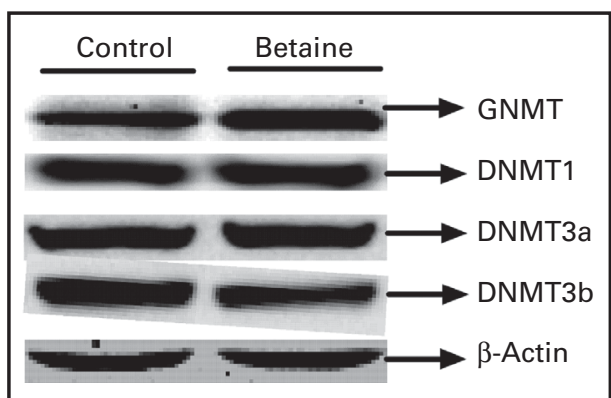

(b)

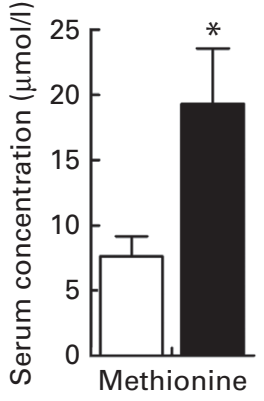

(c)

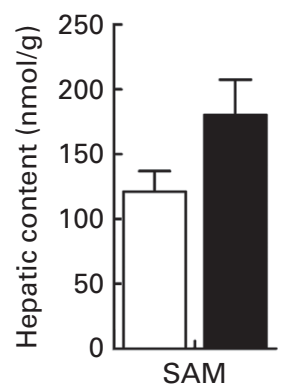

Student's $t$ test with SPSS 17.0 for Windows (SPSS, Inc.). Results from the relative quantification of mRNA, protein, $\mathrm{CpG}$ methylation and miRNA are expressed as the fold change relative to the mean value of the control group. Differences were considered statistically significant at $P<0 \cdot 05$.

\section{Results}

Reproductive performance of sows and body weight and liver weight of piglets

Maternal betaine supplementation did not affect the litter size or litter weight (see online supplementary Table S1). Moreover, newborn piglets born to betaine-supplemented sows exhibited comparable body weight and liver weight to their control counterparts (Table 2)

\section{Hepatic betaine and methionine metabolism}

Piglets born to betaine-supplemented sows had significantly higher hepatic betaine content $(P<0.05$; Fig. 1(a)) and serum methionine concentration $(P<0.05$; Fig. 1(b)). Moreover, hepatic SAM content tended to be higher $(P=0.09)$ in piglets born to betaine-supplemented sows (Fig. 1(c)). These alterations were associated with significantly up-regulated mRNA $(P<0.05$; Fig. $1(\mathrm{~d}))$ and protein $(P<0.05$; Fig. 1(e) and (f)) expression of GNMT. However, no significant alteration was observed for the mRNA or protein expression of DNMT1, DNMT3a $(P=0.06)$ or DNMT3b in the liver of newborn piglets born to betaine-supplemented mothers (Fig. 1(d)-(f))

(d)

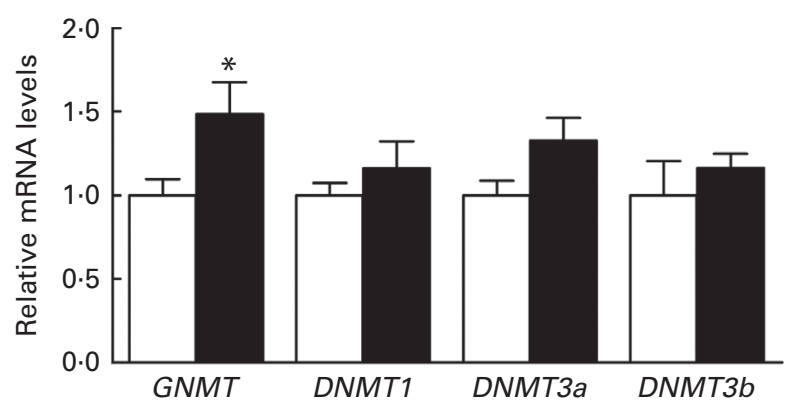

(f)

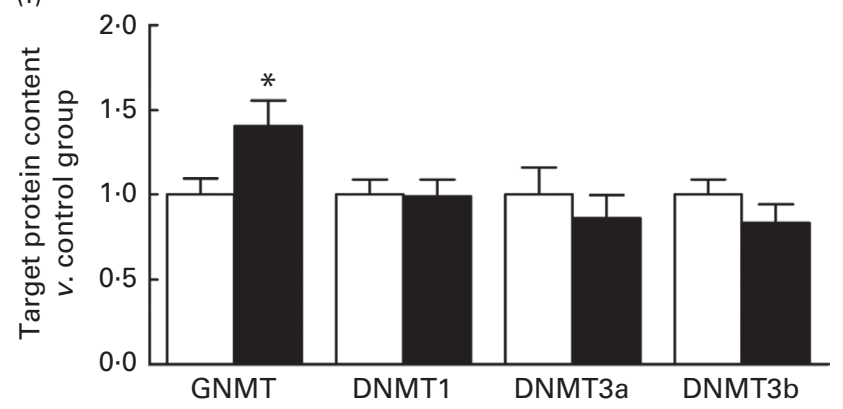

Fig. 1. Hepatic betaine (a), serum methionine concentration (b) and hepatic S-adenosylmethionine (SAM) (c) content, gene (d) and protein (e, f) expression of glycine $N$-methyltransferase (GNMT) and DNA methyltransferases (DNMT) in the liver of newborn piglets. Values are means ( $n$ ), with their standard errors represented by vertical bars. ${ }^{*}$ Mean value was significantly different from that of the control group $(P<0 \cdot 05)$. $\square$, Control group; $\mathbf{\square}$, betaine group. 
(a)

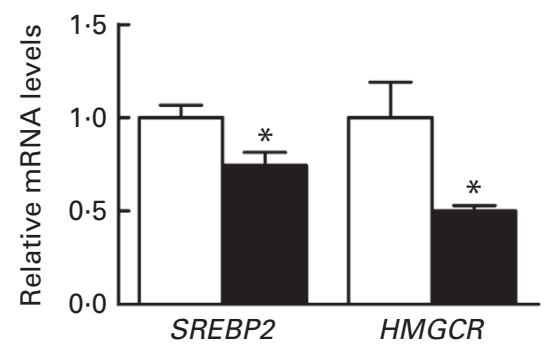

(d)

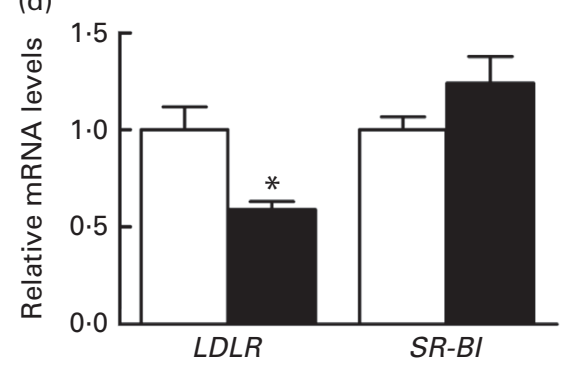

(g)

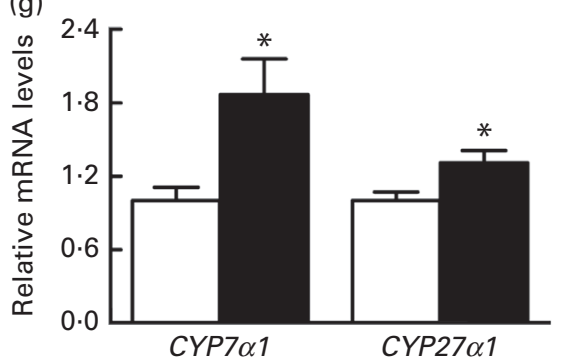

(b)

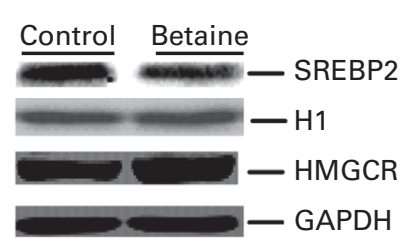

(e)

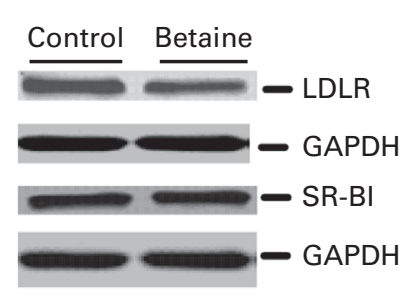

(h)

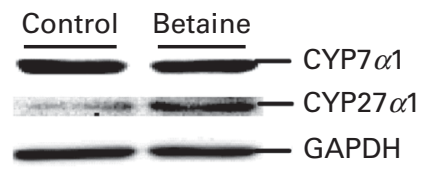

(c)

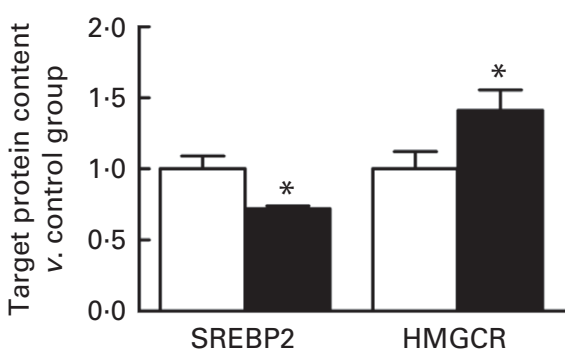

(f)
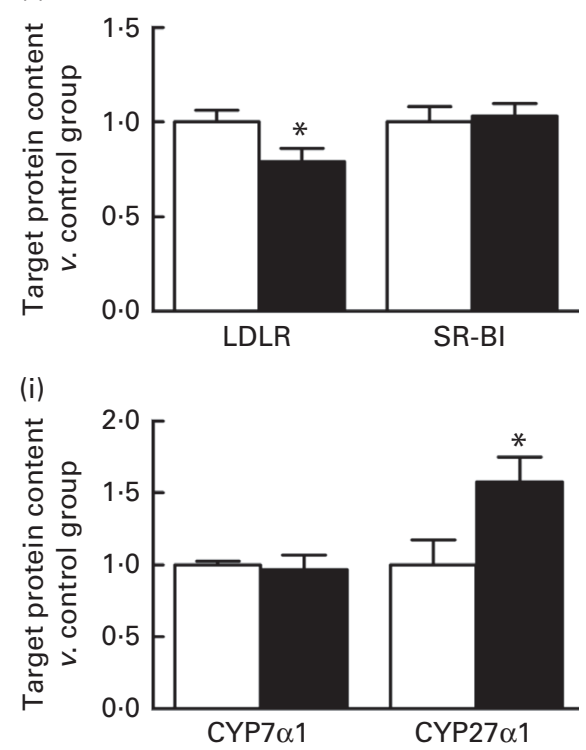

Fig. 2. Hepatic mRNA abundance, Western blot analysis and summary of the protein expression of sterol regulatory element-binding protein-2 (SREBP2) and 3-hydroxy-3-methylglutaryl CoA reductase (HMGCR) in cholesterol biosynthesis $(\mathrm{a}-\mathrm{c})$, those of LDL receptor (LDLR) and scavenger receptor class $\mathrm{B}$ type I (SR-BI) in cholesterol transport and uptake (d-f), and those of cholesterol-7 $\alpha$-hydroxylase (CYP7 $\alpha 1)$ and cholesterol-27 $\alpha$-hydroxylase (CYP27 $\alpha 1)(g-i)$ in cholesterol transformation in the liver of newborn piglets. Values are means $(n 8)$, with their standard errors represented by vertical bars. ${ }^{*}$ Mean value was significantly different from that of the control group $(P<0.05)$. $\mathrm{H} 1$, Histone $\mathrm{H1}$; GAPDH, glyceraldehyde 3-phosphate dehydrogenase. $\square$, Control group; $\mathbf{\square}$, betaine group.

\section{Serum cholesterol concentrations and hepatic total cholesterol content}

No significant alterations were observed in the serum concentrations of LDL-C- and HDL-cholesterol or in the ratio of LDL-C:HDL-cholesterol. However, hepatic content of total cholesterol was significantly increased $(P<0.05)$ in piglets born to betaine-supplemented sows (Table 2).

\section{Hepatic expression of cholesterol metabolic genes}

Among the genes involved in cholesterol de novo synthesis, $H M G C R$ was significantly reduced $(P<0.05)$ by $45 \%$ at the mRNA level in piglets born to betaine-supplemented sows (Fig. 2(a)), which was accompanied by a significant downregulation $(P<0.05)$ of SREBP2 at both mRNA and nuclear protein content levels (Fig. 2(a)-(c)). However, the hepatic protein content of HMGCR was significantly higher $(P<0.05)$ in piglets born to betaine-supplemented sows (Fig. 2(b) and (c)).

Among the genes involved in cholesterol transport and uptake, although maternal betaine supplementation did not alter the serum concentrations of LDL-C or HDL-cholesterol in neonatal offspring (Table 2), hepatic LDLR was significantly down-regulated $(P<0.05)$ at both mRNA (Fig. 2(d)) and protein (Fig. 2(e) and (f)) levels. In contrast, neither the mRNA nor the protein expression of SR-BI was altered in betaineexposed piglets (Fig. 2(d)-(f)).

Of the genes involved in cholesterol transformation, hepatic $C Y P 7 \alpha 1$ mRNA was significantly up-regulated $(P<0.05)$ by $87 \%$ in newborn piglets born to betaine-supplemented sows (Fig. 2(g)), but no alteration was observed at the protein level (Fig. 2(h) and (i)). In contrast, the expression of CYP27 1 was up-regulated $(P<0.05)$, at both mRNA and protein levels, in the liver of piglets born to betainesupplemented sows (Fig. 2(g)-(i)).

\section{Epigenetic modifications of altered cholesterol metabolic genes}

The level of $\mathrm{CpG}$ methylation on the $H M G C R$ promoter was 2.34-fold higher $(P<0.05)$ in the liver of piglets born to betaine-supplemented sows (Fig. 3(a)), which was in parallel with a $40 \%$ increment $(P<0.05)$ of the repressive 
(a)

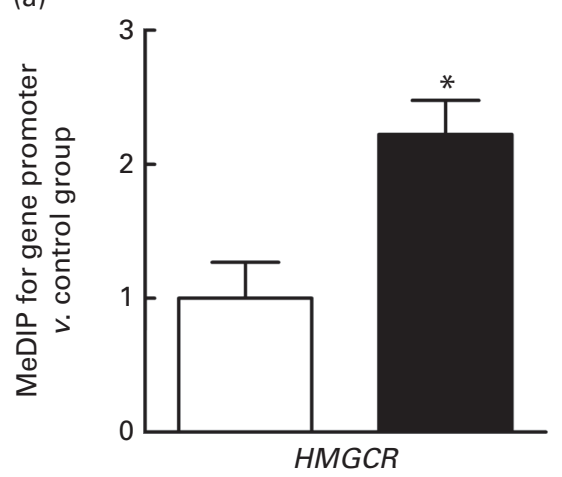

(c)

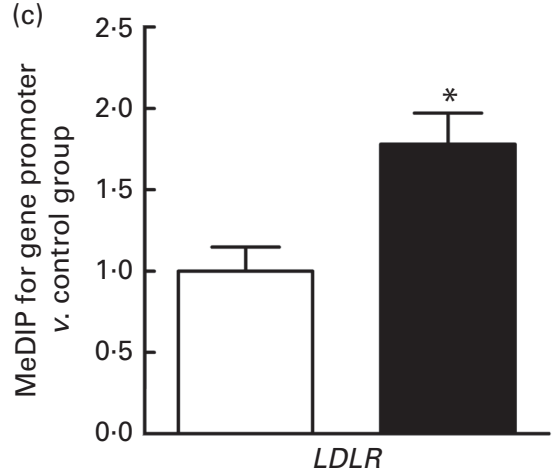

(b)

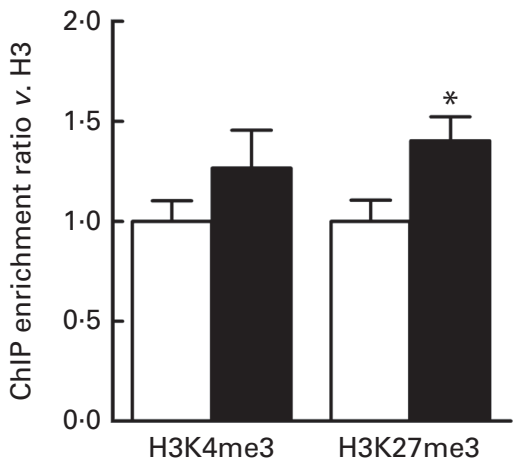

(d)

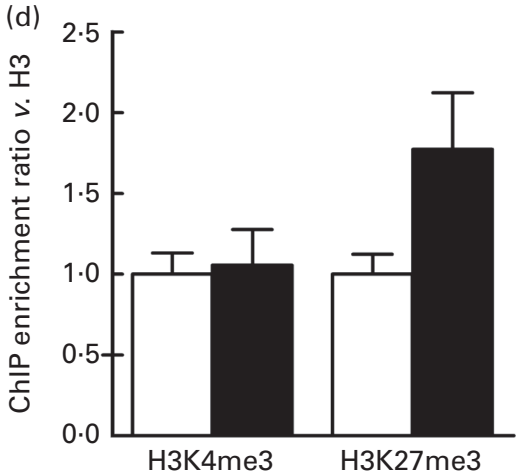

Fig. 3. Methylated DNA immunoprecipitation (MeDIP) and chromatin immunoprecipitation (ChIP) analyses of DNA methylation and histone modifications on the gene promoter of 3-hydroxy-3-methylglutaryl CoA reductase (HMGCR) (a, b) and LDL receptor (LDLR) (c, d) in the liver of newborn piglets. Values are means $(n)$, with their standard errors represented by vertical bars. ${ }^{\star}$ Mean value was significantly different from that of the control group $(P<0.05)$. $\mathrm{H} 3$, Histone $\mathrm{H} 3$; H3K4me3, histone H3 lysine 4 trimethylation; H3K27me3, histone H3 lysine 27 trimethylation. $\square$, Control group; $\mathbf{\square}$, betaine group.

histone mark H3K27me3 (histone H3 lysine 27 trimethylation; Fig. 3(b)). Significant DNA hypermethylation $(P<0.05$; Fig. 3(c)) and a trend of more enriched H3K27me3 mark $(P=0.06$; Fig. 3(d)) was also detected in the promoter of the $L D L R$ gene in the liver of piglets born to betainesupplemented sows. Because no $\mathrm{CpG}$ islands were found within the $5^{\prime}$ flanking sequence of the porcine $C Y P 7 \alpha 1$, $C Y P 27 \alpha 1$ or SREBP2 genes, and the SR-BI mRNA level was not changed in betaine-exposed piglets, we excluded $C Y P 7 \alpha 1, C Y P 27 \alpha 1, S R E B P 2$ and SR-BI genes from methylated DNA immunoprecipitation and chromatin immunoprecipitation analyses in the present study.

\section{Hepatic expression of microRNA targeting altered cholesterol metabolic genes}

Piglets born to betaine-supplemented sows demonstrated a significant down-regulation $(P<0.05)$ in the hepatic expression of miR-497 (Fig. 4(a)), which is predicted to target $H M G C R$. Moreover, LDLR-targeting miRNA, including miR-1285, miR-138, miR-4334-5p and miR-7144-5p, were all up-regulated $(P<0.05)$ in the liver of betaine-exposed piglets (Fig. 4(b)). Furthermore, miR-181 predicted to target $C Y P 7 \alpha 1$ was remarkably up-regulated $(P<0 \cdot 05$; Fig. $4(\mathrm{c}))$, while miR-17-3p, miR-202-3p, miR-30-3p, miR-361-3p and miR-7143-3p predicted to target $C Y P 27 \alpha 1$ were significantly down-regulated $(P<0.05$, Fig. $4(\mathrm{~d}))$ in the liver of piglets born to betaine-supplemented sows.

\section{Functional validation of miR-497 and miR-181}

Ectopic expression of miR-497 significantly suppressed $(P<0.05)$ the luciferase activity of HeLa cells co-transfected with the pGL3-Control/HMGCR luciferase reporter plasmid at both 24 and $48 \mathrm{~h}$ (Fig. 5(a)). Similarly, the luciferase activity of HeLa cells transfected with the pGL3-Control/CYP7 1 reporter plasmid was significantly decreased by co-transfection of pSilencer 3.1 H1-neo miR-181 $(P<0 \cdot 05)$ at both 24 and $48 \mathrm{~h}$ (Fig. 5(b)).

\section{Discussion}

In the present study, maternal betaine supplementation during gestation caused a significant increase in hepatic betaine content in neonatal offspring. In mammals, betaine is used as a substrate for methionine synthesis ${ }^{(32)}$. Dietary betaine supplementation has been shown to increase serum methionine concentrations in human subjects ${ }^{(33)}$. In agreement with these previous findings, we found elevated serum methionine concentrations associated with higher hepatic betaine content in betaine-exposed piglets. Previous observation indicates that excessive methionine leads to an accumulation of SAM and 
(a)

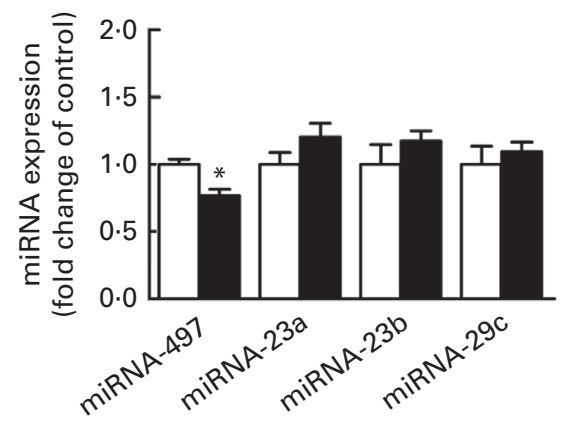

(c)

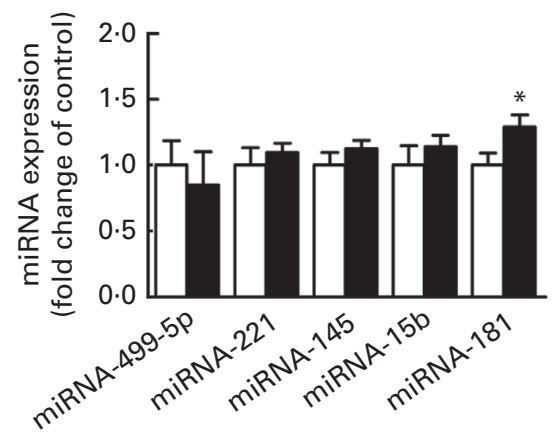

(b)

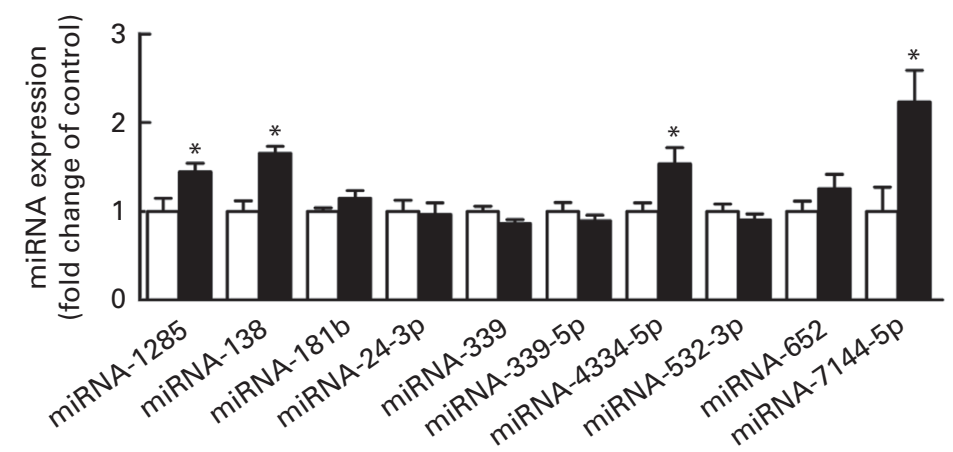

(d)

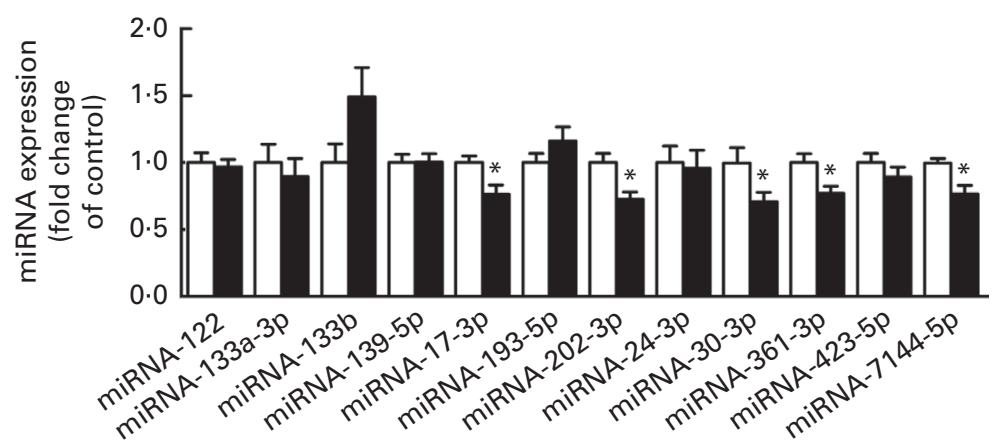

Fig. 4. MicroRNA (miRNA) predicted to target $3^{\prime}$-UTR of 3-hydroxy-3-methylglutaryl CoA reductase (HMGCR) (a), LDL receptor (LDLR) (b), cholesterol$7 \alpha$-hydroxylase $(C Y P 7 \alpha 1)$ (c) and cholesterol-27 $\alpha$-hydroxylase (CYP27 $\alpha 1)$ (d) in the liver of newborn piglets. Values are means $(n 8)$, with their standard errors represented by vertical bars. * Mean value was significantly different from that of the control group $(P<0.05)$.

the activation of $\mathrm{GNMT}^{(34)}$. Indeed, betaine-exposed piglets demonstrated a higher hepatic SAM content and activated GNMT expression. GNMT is known to provide methyl groups for DNA and protein methylation through the conversion of SAM to $S$-adenosylhomocysteine ${ }^{(35)}$. Therefore, maternal betaine supplementation during gestation may modify the methylation of DNA and proteins, especially histones, in the liver of betaine-exposed piglets.

Dietary methionine supplementation has been shown to elevate cholesterol concentrations in the liver and plasma of rats $^{(36)}$. In the present study, neonates born to betainesupplemented dams had $37 \cdot 1 \%$ higher hepatic cholesterol content, associated with altered expression of cholesterol metabolic genes involved in cholesterol biosynthesis, transformation and transportation. To our surprise, we found that mRNA abundances of SREBP2, LDLR and CYP27 21 were in accordance with their respective protein content, while HMGCR and CYP7 1 demonstrated dissociated mRNA and protein levels. The regulation of gene expression is known to occur at different levels, namely transcription, post-transcription and translation. For some genes, the regulation occurs predominantly at the level of transcription. In this case, mRNA abundance usually agrees with the protein content. However, many other genes are also subjected to posttranscriptional and/or translational regulations, and in which case, mRNA abundance and protein content can be uncoupled. mRNA abundance reported herein represent the steady-state mRNA level resulting from a balance between transcription and degradation. Proteins are considered to be the executors of biological functions. However, many proteins, especially enzymes, have to be modified post-translationally to gain biological activities. In the present study, neither mRNA abundance nor protein content is directly relevant to biological functions. The results presented herein only demonstrate that cholesterol metabolic genes are differently regulated at both transcription and/or post-transcription levels in the liver of betaine-exposed piglets.

DNA methylation and histone modifications are recognised as crucial mechanisms involved in the transcriptional regulation of cholesterol metabolic genes. For instance, maternal dietary protein restriction increased hepatic cholesterol content in rat offspring through modulating histone modifications on the $C Y P 7 \alpha 1$ promoter and thus altering the mRNA expression of $C Y P 7 \alpha 1^{(14)}$. Moreover, dietary protein restriction in sows increased hepatic $H M G C R$ expression, which was associated with DNA hypomethylation and lower H3K27me3 on the HMGCR gene promoter ${ }^{(16)}$. In the present study, the promoters of HMGCR and LDLR genes were found to be hypermethylated in the liver of piglets prenatally exposed to betaine, which coincided with the down-regulation of these two genes at the mRNA level. Furthermore, $\mathrm{H} 3 \mathrm{~K} 27 \mathrm{me} 3$ is a repressive histone mark that negatively regulates transcription by promoting a compact chromatin structure $^{(37)}$. The diminished HMGCR and LDLR gene expression was associated with more enriched $\mathrm{H} 3 \mathrm{~K} 27 \mathrm{me} 3$ on the respective gene promoters. Therefore, it appears that increased DNA 

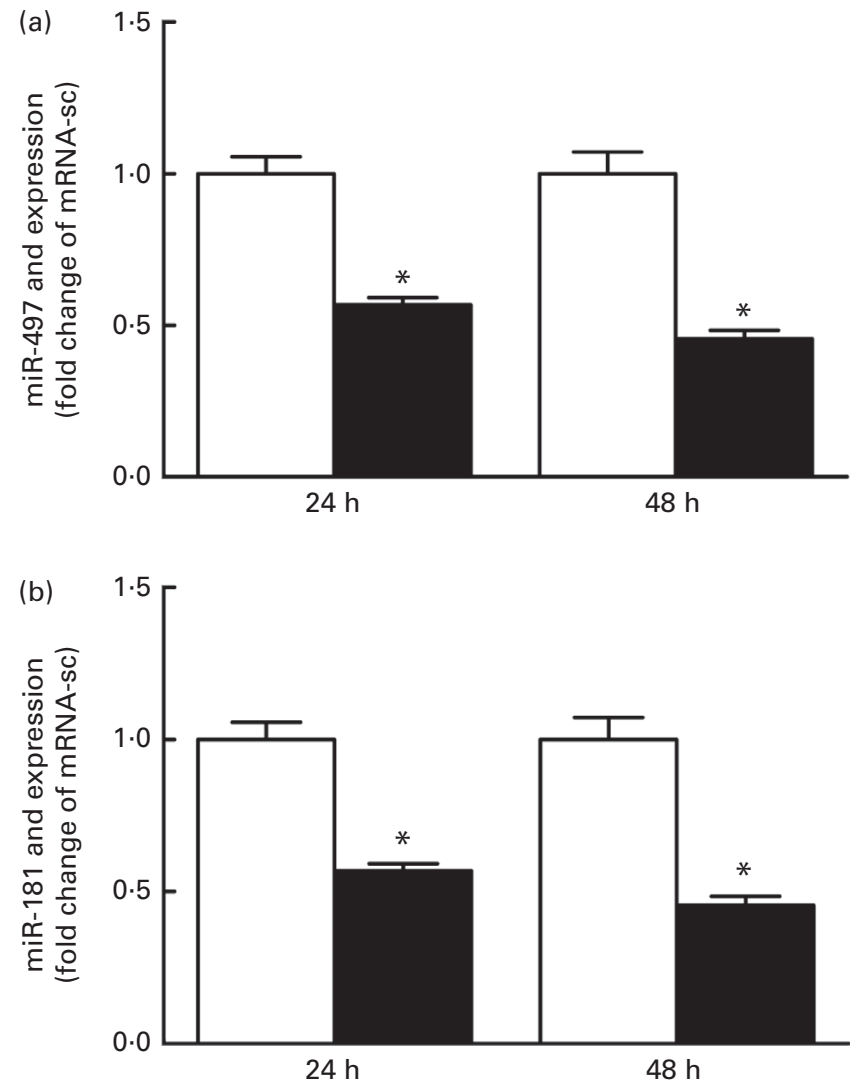

Fig. 5. Validation of ssc-miR-497 (Sus scrofa miR-497) targeting 3-hydroxy3-methylglutaryl CoA reductase (HMGCR) 3 '-UTR (a) and ssc-miR-181 (Sus scrofa miR-181) targeting cholesterol-7 $\alpha$-hydroxylase (CYP7 $\alpha 1$ ) 3'-UTR (b). Values are means $(n 6)$, with their standard errors represented by vertical bars. * Mean value was significantly different from that of the control group $(P<0.05)$. (a) $\square$, miRNA-sc (scrambled control); $\square$, miR-497. (b) $\square$, miRNAsc (scrambled control); $\mathbf{\square}$, miR-181.

methylation and repressive histone mark play a role in the transcriptional regulation of $H M G C R$ and $L D L R$ genes in the liver of piglets in response to prenatal betaine exposure.

It is well known that SREBP-2 is a major transcriptional factor that activates the expression of $H M G C R$ and $L D L R$ genes $^{(10,38)}$. We found a significant down-regulation of SREBP-2 at both mRNA and protein levels in the liver of the betaine-exposed group, which appears to be the direct cause for repressed transcription of $H M G C R$ and $L D L R$ genes. It should be mentioned that the CpG islands on HMGCR and LDLR gene promoters detected in the present study are predicted to contain binding sites for a number of transcriptional factors including SREBP-2 (see online supplementary Fig. S1). This implicates that chromatin remodelling caused by altered DNA and histone methylation status on $H M G C R$ and $L D L R$ promoters may affect the binding of specific transcriptional factors, thereby regulating the transcriptional level of these genes.

Another interesting finding of the present study is the incongruity between the mRNA and protein levels for HMGCR and $C Y P 7 \alpha 1$ genes. $H M G C R$ was decreased at the mRNA level but increased at the protein level, while $C Y P 7 \alpha 1$ was up-regulated at the mRNA level but remained unchanged at the protein level. The dissociation of mRNA abundance and protein content implies the possible involvement of post-transcriptional regulation. MicroRNA are known to participate predominantly in the post-transcriptional regulation through target mRNA degradation and/or translational repression. Previous studies have indicated that miRNA predominantly promotes the cleavage of mRNA in plants ${ }^{(39,40)}$, while miRNA acts mainly through translational repression in animals ${ }^{(41)}$. Moreover, it has been reported that in animal models and cell lines, miRNA targets, in principal, protein translation rather than mRNA degradation ${ }^{(42,43)}$. Therefore, when transcriptional regulation and miRNA-mediated translational repression are not synchronised, mRNA and protein levels can be uncoupled. In the present study, the down-regulated expression of miR-497 was associated with increased protein content of HMGCR, and the up-regulated expression of miR-181 was associated with the unchanged protein level of CYP $7 \alpha 1$, in the liver of betaine-exposed piglets. To verify the targeting sites of miR-497 and miR-181 on 3'-UTR of HMGCR and $C Y P 7 \alpha 1$ transcripts, respectively, we conducted luciferase reporter gene assay and confirmed that miR-497 and miR181 inhibited luciferase activity through targeting $3^{\prime}$-UTR of $H M G C R$ and $C Y P 7 \alpha 1$, respectively.

Betaine insufficiency is associated with the metabolic syndrome including lipid disorder and diabetes, while betaine supplementation increases serum total cholesterol and LDL-C concentrations $^{(25,26,44)}$. Betaine, as a methyl donor, is known to be critical for human embryonic and fetal development ${ }^{(19)}$, and plasma betaine concentration has been reported to be correlated with total cholesterol concentrations in an epidemiological study in human subjects ${ }^{(45)}$. Owing to their similarities to humans in anatomy, body size, physiology, metabolism and omnivorous habits, pigs are better-suited for human metabolic studies when compared with rodents ${ }^{(46,47)}$. Although mostly descriptive, the results presented herein provide the first evidence that maternal dietary betaine supplementation during gestation causes complex and different changes in the hepatic expression of cholesterol metabolic genes in neonatal piglets, with the involvement of epigenetic modifications including DNA and histone methylation, as well as miRNA expression. These findings may help understand the role of maternal betaine supplementation in fetal programming of cholesterol metabolism in humans.

Obviously, the present study has some limitations. First, epigenetic regulation is only one of the possible mechanisms underlying the altered hepatic expression of cholesterol metabolic genes. The participation of other regulatory mechanisms, such as transcriptional factors and their interaction with epigenetic mechanisms, cannot be ruled out. Second, changes in hepatic cholesterol metabolism in neonates may persist to adulthood, causing long-term consequences in cholesterol homeostasis later in adult life. Follow-up studies are necessary to address the long-term effects on adult health and disease in response to the fetal programming of hepatic cholesterol metabolism caused by maternal betaine supplementation. 


\section{Supplementary material}

To view supplementary material for this article, please visit http://dx.doi.org/10.1017/S0007114514002402

\section{Acknowledgements}

The present study was supported by the National Basic Research Program of China (2012CB124703), the Special Fund for Agro-Scientific Research in the Public Interest (201003011), the Fundamental Research Funds for the Central Universities (KYZ200913), the Priority Academic Program Development of Jiangsu Higher Education Institutions and the Innovation Project of Jiangsu Province Postgraduate Education (2013CXLX13_292).

We thank Dr Haoyu Liu (Uppsala University, Sweden) for critical comments on the manuscript.

The authors' contributions were as follows: D. C. performed the experiments and measurements of serum biochemical parameters, analysed and interpreted the results, and drafted the manuscript; Y. J. and J. L. determined the serum hormone and amino acid levels; M. Y., S. S. and H. S. contributed to the experimental design; R. Z. contributed to the experimental concepts and design, provided scientific direction, analysed and interpreted the results, and finalised the manuscript. All authors read and approved the final manuscript.

There are no conflicts of interest to declare.

\section{References}

1. Grundy SM (1978) Cholesterol metabolism in man. West J Med 128, 13-25.

2. Grundy SM (2004) Obesity, metabolic syndrome, and cardiovascular disease. J Clin Endocrinol Metab 89, 2595-2600.

3. Grundy SM, Cleeman JI, Daniels SR, et al. (2005) Diagnosis and management of the metabolic syndrome: an American Heart Association/National Heart. Lung, and Blood Institute Scientific Statement. Circulation 112, 2735-2752.

4. Woollett LA (2005) Maternal cholesterol in fetal development: transport of cholesterol from the maternal to the fetal circulation. Am J Clin Nutr 82, 1155-1161.

5. Roux C, Wolf C, Mulliez N, et al. (2000) Role of cholesterol in embryonic development. Am J Clin Nutr 71, 1270S-1279S.

6. Edison RJ, Berg K, Remaley A, et al. (2007) Adverse birth outcome among mothers with low serum cholesterol. Pediatrics 120, 723-733.

7. Schoknecht PA, Ebner S, Pond WG, et al. (1994) Dietary cholesterol supplementation improves growth and behavioral response of pigs selected for genetically high and low serum cholesterol. J Nutr 124, 305-314.

8. Lu CD, Pond WG, Mersmann HJ, et al. (1995) Response to dietary fat and cholesterol in young adult boars genetically selected for high or low plasma cholesterol. J Anim Sci $\mathbf{7 3}$, 2043-2049.

9. Khovidhunkit W, Kim MS, Memon RA, et al. (2004) Effects of infection and inflammation on lipid and lipoprotein metabolism: mechanisms and consequences to the host. J Lipid Res 45, 1169-1196.

10. Horton JD, Shimomura I, Brown MS, et al. (1998) Activation of cholesterol synthesis in preference to fatty acid synthesis in liver and adipose tissue of transgenic mice overproducing sterol regulatory element-binding protein-2. J Clin Invest 101, 2331-2339.

11. Brown MS \& Goldstein JL (1980) Multivalent feedback regulation of HMG CoA reductase, a control mechanism coordinating isoprenoid synthesis and cell growth. J Lipid Res 21, 505-517.

12. Wyne KL \& Woollett LA (1998) Transport of maternal LDL and HDL to the fetal membranes and placenta of the Golden Syrian hamster is mediated by receptor-dependent and receptor-independent processes. J Lipid Res 39, 518-530.

13. Dias V \& Ribeiro V (2011) Ethnic differences in the prevalence of polymorphisms in CYP7A1, CYP7B1 AND CYP27A1 enzymes involved in cholesterol metabolism. J Pharm Bioallied Sci 3, 453-459.

14. Sohi G, Marchand K, Revesz A, et al. (2011) Maternal protein restriction elevates cholesterol in adult rat offspring due to repressive changes in histone modifications at the cholesterol 7 alpha-hydroxylase promoter. Mol Endocrinol 25, 785-798

15. Goharkhay N, Tamayo EH, Yin HZ, et al. (2008) Maternal hypercholesterolemia leads to activation of endogenous cholesterol synthesis in the offspring. Am J Obstet Gynecol 199, $273 \mathrm{e} 1-273 \mathrm{e} 6$.

16. Cong RH, Jia YM, Li RS, et al. (2012) Maternal low-protein diet causes epigenetic deregulation of HMGCR and CYP $7 \alpha 1$ in the liver of weaning piglets. J Nutr Biochem 23, 1647-1654.

17. Weaver IC, Champagne FA, Brown SE, et al. (2005) Reversal of maternal programming of stress responses in adult offspring through methyl supplementation: altering epigenetic marking later in life. J Neurosci 25, 11045-11054.

18. Lillycrop KA, Rodford J, Garratt ES, et al. (2010) Maternal protein restriction with or without folic acid supplementation during pregnancy alters the hepatic transcriptome in adult male rats. Br J Nutr 103, 1711-1719.

19. Lever M \& Slow S (2010) The clinical significance of betaine, an osmolyte with a key role in methyl group metabolism. Clin Biochem 43, 732-744.

20. Ji Y, Nordgren KKS, Chai YB, et al. (2012) Human liver methionine cycle: MAT1A and GNMT gene resequencing, functional genomics, and hepatic genotype-phenotype correlation. Drug Metab Dispos 40, 1984-1992.

21. Bestor TH (2000) The DNA methyltransferases of mammals. Hum Mol Genet 9, 2395-2402.

22. Cordero P, Gomez-Uriz AM, Campion J, et al. (2013) Dietary supplementation with methyl donors reduces fatty liver and modifies the fatty acid synthase DNA methylation profile in rats fed an obesogenic diet. Genes Nutr 8, 105-113.

23. Eklund M, Bauer E, Wamatu J, et al. (2005) Potential nutritional and physiological functions of betaine in livestock. Nutr Res Rev 18, 31-48.

24. Ratriyanto A, Mosenthin R, Bauer E, et al. (2009) Metabolic, osmoregulatory and nutritional functions of betaine in monogastric animals. Asian Aust J Anim Sci 22, 1461-1476.

25. McGregor DO, Dellow WJ, Robson RA, et al. (2002) Betaine supplementation decreases post-methionine hyperhomocysteinemia in chronic renal failure. Kidney Int 61, 1040-1046.

26. Schwab U, Torronen A, Toppinen L, et al. (2002) Betaine supplementation decreases plasma homocysteine concentrations but does not affect body weight, body composition, or resting energy expenditure in human subjects. Am J Clin Nutr 76, 961-967.

27. Yang HS, Lee JI, Joo ST, et al. (2009) Effects of dietary glycine betaine on growth and pork quality of finishing pigs. Asian Aust J Anim Sci 22, 706-711. 
28. Martins JM, Neves JA, Freitas A, et al. (2010) Betaine supplementation affects the cholesterol but not the lipid profile of pigs. Eur J Lipid Sci Technol 112, 295-303.

29. Kirsch SH, Herrmann W, Rabagny Y, et al. (2010) Quantification of acetylcholine, choline, betaine, and dimethylglycine in human plasma and urine using stable-isotope dilution ultra performance liquid chromatography-tandem mass spectrometry. J Chromatogr B Analyt Technol Biomed Life Sci 878, 3338-3344.

30. Liu X, Wang J, Li R, et al. (2011) Maternal dietary protein affects transcriptional regulation of myostatin gene distinctively at weaning and finishing stages in skeletal muscle of Meishan pigs. Epigenetics 6, 899-907.

31. Kertesz M, Iovino N, Unnerstall U, et al. (2007) The role of site accessibility in microRNA target recognition. Nat Genet 39, $1278-1284$.

32. Dominguez-Salas P, Moore SE, Cole D, et al. (2013) DNA methylation potential: dietary intake and blood concentrations of one-carbon metabolites and cofactors in rural African women. Am J Clin Nutr 97, 1217-1227.

33. Storch KJ, Wagner DA \& Young VR (1991) Methionine kinetics in adult men: effects of dietary betaine on 1-[ $\left[{ }^{2} \mathrm{H}_{3}\right.$-methyl-1- $\left.{ }^{13} \mathrm{C}\right]$ methionine. Am J Clin Nutr 54, 386-394.

34. Varela-Rey M, Martinez-Lopez N, Fernandez-Ramos D, et al. (2010) Fatty liver and fibrosis in glycine $N$-methyltransferase knockout mice is prevented by nicotinamide. Hepatology $\mathbf{5 2}$, 105-114.

35. Cook RJ \& Wagner C (1984) Glycine $N$-methyltransferase is a folate binding protein of rat liver cytosol. Proc Natl Acad Sci US A 81, 3631-3634.

36. Hirche F, Schroder A, Knoth B, et al. (2006) Effect of dietary methionine on plasma and liver cholesterol concentrations in rats and expression of hepatic genes involved in cholesterol metabolism. Br J Nutr 95, 879-888.
37. Ringrose L, Ehret H \& Paro R (2004) Distinct contributions of histone $\mathrm{H} 3$ lysine 9 and 27 methylation to locus-specific stability of polycomb complexes. Mol Cell 16, 641-653.

38. Goldstein JL \& Brown MS (1990) Regulation of the mevalonate pathway. Nature 343, 425-430.

39. Kawasaki H \& Taira K (2004) MicroRNA-196 inhibits HOXB8 expression in myeloid differentiation of HL60 cells. Nucleic Acids Symp Ser (Oxf) 211-212.

40. Moxon S, Jing R, Szittya G, et al. (2008) Deep sequencing of tomato short RNAs identifies microRNAs targeting genes involved in fruit ripening. Genome Res 18, 1602-1609.

41. Williams AE (2008) Functional aspects of animal microRNAs. Cell Mol Life Sci 65, 545-562.

42. Pan S, Zheng Y, Zhao R, et al. (2013) MicroRNA-130b and microRNA-374b mediate the effect of maternal dietary protein on offspring lipid metabolism in Meishan pigs. $\mathrm{BrJ}$ Nutr 109, 1731-1738.

43. Sangokoya C, Doss JF \& Chi JT (2013) Iron-responsive miR-485-3p regulates cellular iron homeostasis by targeting ferroportin. PLOS Genet 9, e1003408.

44. Rabinowitch IM (1936) Effects of betaine upon the cholesterol and bilirubin contents of blood plasma in diabetes mellitus. Can Med Assoc J 34, 637-641.

45. Ross AB, Bruce SJ, Blondel-Lubrano A, et al. (2011) A wholegrain cereal-rich diet increases plasma betaine, and tends to decrease total and LDL-cholesterol compared with a refinedgrain diet in healthy subjects. Br J Nutr 105, 1492-1502.

46. Miller ER \& Ullrey DE (1987) The pig as a model for humannutrition. Annu Rev Nutr 7, 361-382

47. Neeb ZP, Edwards JM, Alloosh M, et al. (2010) Metabolic syndrome and coronary artery disease in Ossabaw compared with Yucatan swine. Comp Med 60, 300-315. 